\title{
Conflictos de intereses en publicaciones biomédicas
}

\section{Conflicts of interests in biomedical publications}

\author{
María Nelly Niño de Arboleda • Bogotá, D.C. (Colombia)
}

Acta Médica Colombiana, AMC, como órgano de comunicación de la Asociación Colombiana de Medicina Interna, ACMI ${ }^{\circledR}$, acoge los requisitos internacionales relacionados con los conflictos de intereses ocultos que se presentan con alguna frecuencia en las publicaciones biomédicas, los cuales están explícitos en las indicaciones para los autores en las primeras páginas de la revista.

El editor general de AMC en su artículo "El toque de Midas" (1), hace su declaración de conflictos de intereses y resume en forma concisa, clara y honesta el trabajo editorial que involucra a los demás editores, revisores, autores y a todo el personal que trabaja en AMC para conservar no solo la calidad de los artículos publicados sino la confianza de la comunidad médica, nuestros asociados, el Estado, los lectores, los pacientes y, en general, de la sociedad, en una revista independiente y veraz.

Lo anterior se logra con reglas claras y abiertas, de fácil consulta, que son indispensables en la investigación y en la publicación del conocimiento profesional, para lo cual la revista acepta los comentarios bien intencionados en conservar la confianza y la integridad científica.

\section{Conflictos en publicaciones biomédicas.}

El término "conflicto" se deriva del vocablo latino conflictus, que significa "choque", "colisión". En este caso, es el choque entre dos intereses: la integridad científica y un interés personal o financiero.

Este no es concepto nuevo y las publicaciones médicas son susceptibles de conflictos de diferente orden. Molière, en su obra "La malade imaginaire", satiriza la relación entre el doctor y el boticario que explotan a una hipocondríaca para su propio beneficio económico (2). Después de la segunda guerra mundial, los científicos se preocuparon por los conflictos en la búsqueda del avance en la ciencia y se establecieron los primeros controles para evitar que se presentaran conflictos de intereses en la manera de investigar. En los años 70, la mayor parte de la investigación era financiada por los gobiernos y la academia inició la política de control de conflictos de intereses (9).

Según el International Committee of Medical Journal Editors, ICMJE, el conflicto de intereses existe cuando autores, instituciones, revisores o editores tienen relaciones personales o financieras que lleven a engaño, parcialidad o a conductas inapropiadas o que alteren su juicio científico en el manejo editorial. Más adelante se analizan los conflictos de intereses de la industria farmacéutica en las publicaciones médicas.

Basados en la integridad de la investigación y en sus resultados, la comunidad médica exige que la publicación tenga la credibilidad y la transparencia suficiente en sus procesos para el beneficio de sus lectores y pacientes; por esta razón, se han diseñado varias reglas a nivel internacional con la academia, la Organización Mundial de la Salud y los editores de las revistas más prestigiosas desde la declaración inicial de Vancouver que luego dio paso al ICMJE. Los editores de revistas se reúnen periódicamente, analizan y actualizan las recomendaciones para considerar los diferentes aspectos y recomendar la declaración de los conflictos de intereses de las distintas especialidades y disciplinas (químicos, inves-
María Nelly Niño de Arboleda, MD FACP: Editora Asociada Acta Médica Colombiana. Bogotá, D.C. (Colombia).

E-mail: mnarboleda@gmail.com 
tigadores, cardiólogos, ortopedistas, oncólogos y psiquiatras, entre otros) $(3,4)$

En 1997, la declaración de conflictos de intereses solo era del $16 \%$ en todas las revistas de las diferentes especialidades (5). El incremento de la declaración de los conflictos de intereses ha sido gradual, más usada por las revistas que forman parte del ICMJE. En un análisis reciente de 262 revistas, se encontró que el $85 \%$ tenían instrucciones para la declaración de los conflictos de intereses. En octubre de 2009, el ICMJE propuso un formato electrónico para la declaración de los conflictos de intereses $(6,7)$.

En forma similar, el Consortium of Laboratory Medicine Journal Editors establece los lineamientos y políticas de las publicaciones derivadas de la investigación y cuenta con el apoyo de la World Association of Medical Editors, WAME, $\mathrm{y}$ del Comittee on Publication Ethics, COPE. La estrecha relación entre la industria y la academia es una práctica usual entre la comunidad médica dedicada a la investigación, que produce resultados que pueden ser benéficos en el diagnóstico y las nuevas modalidades terapéuticas con resultados innovadores que mejoran la atención médica (10).

\section{Interacciones editoriales con la industria farmacéutica o biomédica}

Las relaciones o interacciones entre la comunidad médica y científica con la industria son comunes, muy variadas e indispensables. Van desde la recepción de un esfero promocional hasta compromisos más profundos, que incluyen honorarios, apoyo financiero en la investigación, compromisos que si no son abiertos al conocimiento público, se pueden prestar para conductas incorrectas tanto en su labor profesional como en la manipulación de los resultados de la investigación en favor de los intereses de la industria, la formulación inadecuada o la labor de "autores fantasma" con engaño en las publicaciones.

En la literatura médica hay ejemplos de estas acciones como el caso informado en Blood de enero de 2009, donde los editores relatan como uno de los revisores se intrigó porque el autor, un prominente investigador, dio los agradecimientos a un empleado de la industria interesada y en la aclaración se encontró que este había suministrado toda la información necesaria para la publicación. Los editores, Dunbar y Tallman, titularon "Ghostbusting at Blood" para denunciar lo ocurrido y el manuscrito fue rechazado. Así es como se preserva la transparencia y la credibilidad de una revista (8). La recomendación es que el grado de participación de la industria se explique y sea transparente. Este conflicto se conoce como "autor fantasma" o "escritor fantasma" cuando no está incluido entre los autores del artículo a pesar de su participación en el mismo.

El trabajo editorial es intenso y contamos con revisores que desarrollan estrategias para mantener la calidad de AMC, para lo cual la información en línea es consultada con preguntas y palabras que han permitido conocer la autenticidad de los escritos recibidos y hacemos todo lo posible para que los lectores reciban una información tan imparcial como sea posible y que contenga una declaración de intereses clara para evitar los conflictos y el fraude (11).

\section{La industria y el apoyo financiero en los estudios de investigación}

Se ha cuestionado la participación y financiación de la industria en la investigación, sin embargo, cada vez es más demandante y complejo mantener los estándares de calidad exigidos de ella, razón por la cual se aumentan los costos y el papel de los patrocinios que garantizan la viabilidad de los proyectos ha llegado a ser crítico. No obstante, las fuentes de financiación pueden afectar a los investigadores y generar conflictos de intereses en sus acciones o juicios, en especial, en la interpretación de los resultados se pueden aumentar los conflictos si el que financia desea alguna ganancia del informe. Este es el aspecto malo o el conflicto.

Los beneficios de la investigación son amplios y trascienden a los pacientes, permiten que los doctores los cuiden mejor y, asimismo, prescripciones más acertadas basadas en los adelantos farmacéuticos o tecnológicos que se derivan de los estudios innovadores que tuvieron patrocinio.

Las asociaciones médicas han recomendado en sus publicaciones la declaración de conflictos de intereses igualmente en las disciplinas con uso de tecnologías.

La función principal de las asociaciones es servir a los intereses de sus miembros, brindar educación médica mediante congresos y publicaciones, y mantener la defensa de la profesión-disciplina y sus pacientes. Son la cara pública de las diferentes profesiones o especialidades y les está permitido recibir patrocinios y promover reuniones educativas.

¿Cuáles son los enfoques más importantes para evitar que surjan conflictos de intereses en su relación con la industria y mantener la integridad en sus publicaciones, programas de educación y demás productos (conferencias, investigación patrocinada)?

Se sugiere iniciar con la declaración de conflictos de intereses de sus asociados en publicaciones y presentaciones (12).

¿Qué enfoque razonable se debe tomar para hacer cumplir las guías sobre los conflictos de intereses?

En primer lugar, seguir el código de ética; en segundo, el seguimiento del cumplimiento por parte de los líderes de la asociación, el manejo trasparente de los dineros de los patrocinadores y un mercadeo apropiado por parte de la industria en las reuniones de educación continua.

Es importante resaltar que durante el entrenamiento de las especialidades, el estudiante no recibe educación sobre los riesgos de conflictos de intereses en su profesión, en la investigación, en la protección de los sujetos que participan en la investigación con humanos o con animales o en la retribución económica que puede derivarse de la comercialización de la propiedad intelectual. Por el contrario, es bien visto que los investigadores estén libres del interés económico que se deriva de los resultados de sus investigaciones. 
Las relaciones industria-asociaciones médicas no necesariamente son malas ni presentan conflictos de intereses siempre y cuando tanto la industria como las organizaciones y sus asociados valoren los riesgos y beneficios, les den un manejo apropiado y mantengan la transparencia de las relaciones. Si los conflictos de intereses se pasan por alto o son ignorados, la confianza de los miembros y la comunidad se pierde causando un daño irreparable.

Históricamente, los avances derivados de la investigación sirven para mejorar y comercializar nuevas terapias. Tal es el caso de Waksman y su grupo: a mediados de 1940 descubrieron un antibiótico, la estreptomicina, con acción sobre el bacilo de la tuberculosis. Waksman hizo que su descubrimiento fuera un aporte mundial y en colaboración con Merck -que produjo el medicamento apto para uso humano-demostró su efectividad en el tratamiento de la tuberculosis mediante un trabajo de asignación aleatoria con gran número de pacientes que hizo la British Medical Association y fue publicado en el British Medical Journal en 1948.

Esta asociación entre un investigador académico, una organización médica y una industria farmacéutica produjo un gran alivio a la humanidad que sufría de tuberculosis y se estableció un modelo todavía vigente hoy en día. Hubiera sido desafortunado si no hubiera ocurrido (14).

Un trabajo convincente ha sido el de la búsqueda de la cura contra el virus del ébola, con la colaboración de la academia, la industria, los pacientes, las organizaciones no gubernamentales y los patrocinadores; otros trabajos han sido esperanzadores en HIV, malaria y en enfermedades no infecciosas como el cáncer, las enfermedades cardiacas, el EPOC, la obesidad y la diabetes (15). El interés es genuino para el bien de la humanidad y de cada uno de los participantes si se conserva el respeto entre ellos, los pacientes y la responsabilidad médica con el compromiso social de controlar y tratar males que afectan a la humanidad.

No es malo ni representa conflicto de intereses, el trabajo individual o en colaboración siempre que haya beneficio para el paciente y no sea para el bien propio $(16,17)$.

En resumen, en un conflicto hay dos partes que chocan: un interés personal oculto (político, ético, de prestigio, institucional, local) o financiero de una de las partes. En el caso de las publicaciones médicas, el conflicto es el choque de la integridad científica contra una voluntad personal o financiera.

La información es importante y las publicaciones médicas son la forma de comunicarla, escrita o por internet (amplia y poco controlada por este medio a veces); la mayoría de las asociaciones científicas expresan en sus revistas lo relacionado con su profesión y es la profesionalidad la base de la defensa de los grupos, entendiendo que el profesionalismo médico compete a los compromisos de la profesión, en su ética, integridad, trasparencia y confianza que el grupo debe conservar ante el resto de la comunidad médica, los pacientes y la sociedad $(18,19)$.
Y, ¿cómo mantener esta integridad y autonomía médica? ¿Qué se enseña al respecto durante la formación del estudiante? ¿Es suficiente la revisión por pares en el esfuerzo editorial de trasparencia? ¿Es suficiente la declaración de conflictos de intereses por parte de los autores y editores para mantenerla? ¿Qué esperan los editores?

Entre las respuestas encontramos que el profesionalismo, la ética y las reglas claras son la base para la independencia, seguir el juramento sobre hacer el bien a los pacientes y mantener la credibilidad, autonomía y confianza de los lectores y pacientes. Además, contribuir a un manejo de costos en salud racionales, consideración con la sociedad (20-22).

Los editores tienen como objetivo de una buena publicación científica que sea íntegra, honesta y transparente, que los artículos se ajusten a las normas claras de publicación, una buena práctica en publicación científica para generar y mantener la confianza de sus lectores.

\section{Referencias}

1. Matijasevich E. El toque de Midas. Acta Med Colomb 2010;35:37-9.

2. Luscher TF. Good publishing practice. Eur Heart J 2012;33:557-61.

3. De Angelis CD, Fontanarosa PB. Resolving unreporting conflicts of interest. JAMA. 2009;302:198-9.

4. Drazen JM, Laine C, De Angelis CD, et al. Toward more uniform conflict disclosures: The updated ICMJE conflict of interest reporting form. $N$ Engl J Med. 2010;363:188-9.

5. Krimsky S, Rothenberg LS. Conflict of interest policies in science and medical journals: Editor's practice and author's disclosures. Sci Eng Ethics. 2001;7:205-18.

6. Ancker JS, Flanagin A. A comparison of conflict of interest policies at peerreviewed journals in different scientific disciplines. Sci Eng Ethics. 2007;13:147-57.

7. Alonso F, et al. Conflicts of interest policies and disclosure requirements among European Society of Cardiology national cardiovascular journals. Cir J. 2012;76:1542-9

8. Dunbar C, Tallman MS. Ghostbusting at Blood. Blood. 2009;113:502-3.

9. Parascandola M. A turning point for conflicts of interest: The controversy over the National Academy of Science's first conflict of interest disclosure policy. $J$ Clin Oncol. 2007;25:3774-9.

10. Nader R. Full disclosure in industry-sponsored Laboratory Medicine Research Studies: Statement by the Consortium of Laboratory Medicine Journal Editors. Clin Chem. 2011;57:359-60.

11. Comité Editorial. Indicaciones a los autores. Acta Med Colomb 2015; 40: II-VI (número 2).

12. Camilleri M, Parke DM. Perspective: Conflict of interest and professional organizations: Considerations and recommendations. Acad Med. 2010;85:85-91.

13. Rotman DJ, McDonald WJ, Berkowist CD, et al. Relations with the industry a proposal for controlling conflicts of interest. JAMA. 2009;301:1367-72.

14. Drazen JM. Revisiting the commercial-academic interface. N Engl J Med. 2015; 372:1853-54.

15. Rosenbaum L. Reconnecting the dots: Reinterpreting industry-physicians relations. N Engl J Med. 2015;372:1860-4

16. DeAngelis CD. Conflicts of interest in medical practice and their costs to the Health Care System. Milbank Q. 2014;92:196-8.

17. Hirsch LJ. Conflicts of interest, authorship and disclosures in industry-related scientific publications the tort bar editorial oversight of medicals journals. Mayo Clin Proc. 2009;84:811-21.

18. Lenier W. Bidirectional conflicts of interest involving industry and medical journals: Who will be the champion integrity? Mayo Clin Proc. 2009;84:771-5.

19. Luscher TF. Good publishing practice. Eur Heart J. 2012;33:557-61.

20. Rosenbaum L. Beyond moral outrage weighting the trade-offs of COI regulation. N Engl J Med. 2015;372:2064-8.

21. Trust, but verify. Nature. 2009;7262:315.

22. Siegler M. Training doctors for professionalism. Mt Sinai J. 2002;69:404-9. 